\title{
Lung Cancer Presented as Skin Metastases: A Case Report
}

Kumar $\mathbf{A}^{*}$ and Aggarwal $\mathbf{P}$

Department of Pulmonary Medicine, Government Medical College and Hospital, Chandigarh, India

\begin{abstract}
Lung cancer with skin metastasis is rare entity and associated with poor prognosis. It can easily lead to misdiagnosis and mistreatments when it presents with skin involvement, so early recognition of the skin metastasis is important as it may be the presenting sign of an underlying malignancy. Most common sites for skin metastasis are chest, back, abdomen, head, and neck and they are usually single or multiple painless nodules. However, the skin metastasis in squamous and small cell lung carcinoma is uncommon and it carries rapidly fatal clinical outcome. We present a rare case of non-small cell lung cancer presenting as rapidly growing skin metastasis.
\end{abstract}

Keywords: Skin metastases; Lung cancer; Squamous cell carcinoma

Abbreviations: CT: Computed Tomography; CXR: Chest X-Ray; LCC: Large-Cell Carcinoma; LDH: Lactate Dehydrogenase; SCC: Squamous-Cell Carcinoma, Usg: Ultrasound

\section{Background}

Skin metastases in non-small cell carcinoma are rare, with incidence of $1.3 \%$ to $7.9 \%$ in various studies [1-3]. They represent about $1 \%$ of all cases of metastatic disease and prognosis is usually poor [1]. Skin metastasis from lung cancer is rare than all other organs and it rapidly progress to other organs leading early death during or before the course of treatment $[4,5]$. Squamous cell carcinoma manifesting with cutaneous metastases is most often from the oral cavity and lungs and less commonly from the uterine cervix, penis or a distant skin site. Previous study showed that adenocarcinoma histology most commonly metastasize in the skin in lung cancer patients [1]. Skin lesions in lung cancer patient can be the first sign of malignancy and it does not have any specific presentation which makes it more difficult to diagnosed correctly which may delay treatment [6]. Skin metastases most commonly seen on chest abdomen head and neck but can occur at any site. They usually occur as round or oval nodules or plaque-like, mobile or fixed, firm, pink, skin-colored and rarely appear as papules, zosteriform, erysipelas-like or as cicatricial alopecia on the scalp [6-12]. We present a rare case of non-small cell lung cancer presented with early rapidly growing skin metastases.

\section{Case Presentation}

A 40-year-old non-smoker, male was admitted to our department with multiple skin papules localized in the right anterior chest and dyspnea on exertion since two months. The papules were soft, zosteriform, erysipelaslike tender, skin-colored and measured 20-22 millimeters in greatest dimension (Figure 1). The patient had history of significant weight loss (>8 killograms in 2 months), anorexia and fatigue. Initialy patient was treated as herpes zoster virus infection with antiviral and antibiotics outside but patient did not improved with same. Patient was sent for excisional biopsy of the lesions in dermatology ward. On histopathology it showed metastatic lesion, likely squamouscell carcinoma. On chest X-ray (CXR) there was homogeneous opacity in the right middle and lower zones with left tracheal shift (Figure 2). Computed tomography (CT) revealed heterogeneously enhancing soft tissue density with multiple loculated fluid collections in between and right para-tracheal and mediastinal lymphadenopathy (Figure 3). Ultrasound (Usg) abdomen and prostate was done to rule out distant metastasis which was normal. Ultrasound chest done which suggested multiple septations with loculated fluid collection in between the mass like consistency (Figure 4). Ultrasound guided fluid aspiration and true

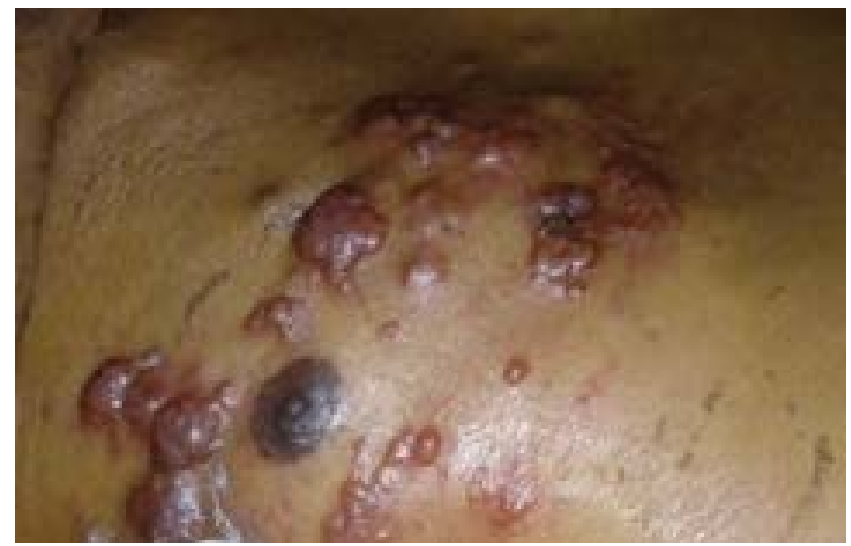

Figure 1: Shows, plaque-like, zosteriform, papular lesion measured 20-22 millimeters in greatest dimension.

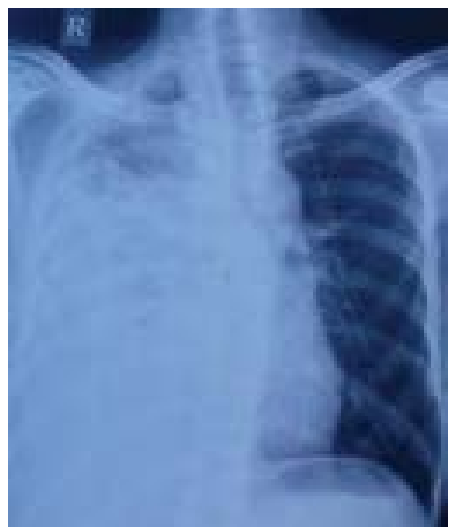

Figure 2: Chest $\mathrm{X}$-ray showing homogeneous opacity in the right middle and lower zones with left tracheal shift.

*Corresponding author: Arjun Kumar, Department of Pulmonary Medicine Government Medical College and Hospital, Chandigarh, India, Tel: 8054809188; E-mail: aknlnegi@gmail.com

Received August 30, 2018; Accepted September 06, 2018; Published September 13, 2018

Citation: Kumar A, Aggarwal P (2018) Lung Cancer Presented as Skin Metastases: A Case Report. J Pulm Respir Med 8: 470. doi: 10.4172/2161-105X.1000470

Copyright: @ 2018 Kumar A, et al. This is an open-access article distributed under the terms of the Creative Commons Attribution License, which permits unrestricted use, distribution, and reproduction in any medium, provided the original author and source are credited. 


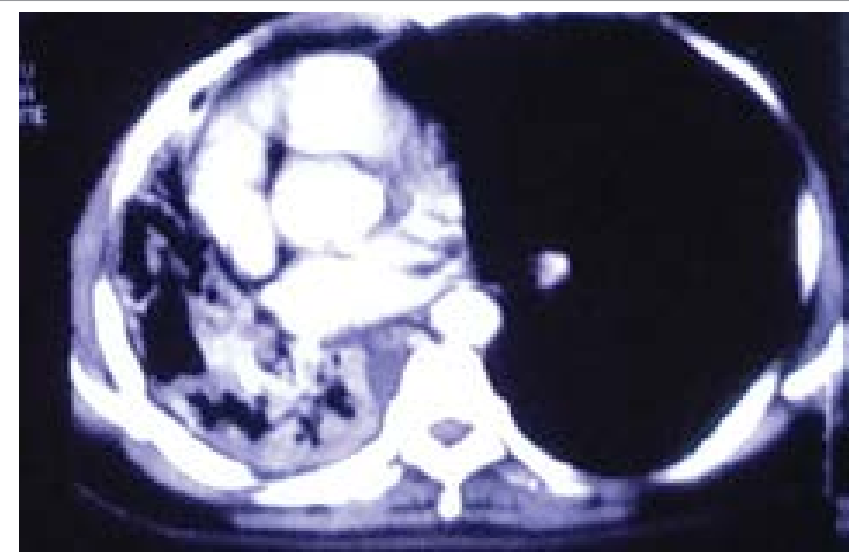

Figure 3: Computed tomography (CT) showing heterogeneously enhancing soft tissue density with right hilar, middle and lower lobes.

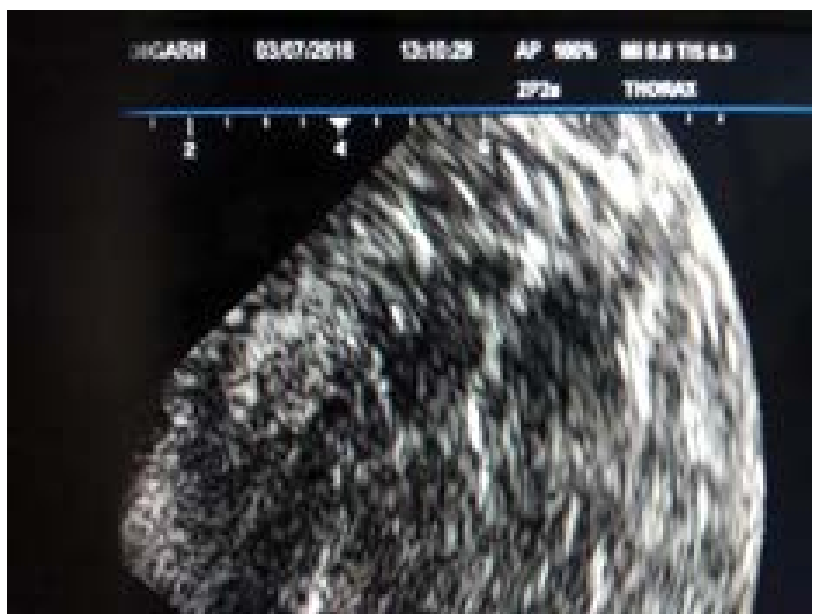

Figure 4: Usg chest showing multiple septations with loculations fluid collection.

cut biopsy was done and cytology revealed malignant cells positive; whereas, histopathology revealed poorly differentiated carcinoma consistent with squamous cell carcinoma. Immunohsitochemical (IHC) pattern analysis was done which revealed p63, cytokeratin $5 / 6$ antibodies (CK5/6) positive and thyroid transcription factor 1( TTF1) was negative. Baseline blood investigation results were within normal limits, except elevated values of lactate dehydrogenase (LDH) 675 $\mathrm{U} / \mathrm{l}$ (230-460) and low serum albumin level $2.1 \mathrm{gm} / \mathrm{dl}$. The oncology openion regarding chemotherapy was taken where they advised palliative chemotherapy with cisplatin $150 \mathrm{mg}$ and etoposide $200 \mathrm{mg}$. But within three weeks of chemotherapy patient developed increased shortness of breath and massive right side pleural effusion. Ultrasound guided multiple therapeutic pleural fluid aspirations was done to relieve progressive dyspnea. Unfortunately, patient passed away one month after the two cycles of chemotherapy.

\section{Discussion}

Metastases skin cancer in lung cancer are rare and presence of skin metastases as the initial presentation of lung cancer is associated with poor prognosis [3]. Skin metastasis is the common site (25\%) of lung cancer and it suggest worse prognosis and rapid progression of disease. The skin metastasis in patient with lung cancer is poor prognostic marker and early skin biopsy is extremely important in such type patients [8]. The definitive diagnosis can be made by a biopsy of the skin lesion with histological analysis and the surgical resection and or radiation therapy is treatment of choice. The mechanisms determining the metastasis of lung cancer in skin is still unknown. Adenocarcinoma of the lung shows the most common tendency to metastasize to the skin, followed by squamous cell carcinoma and large cell carcinoma respectively [9].

In our case, the primary cancer was lung cancer with histology of squamous cell type located on the right middle and lower lobes. Skin metastases were the first sign in our case present on right anterior chest. Although the literature describes cases where metastatic nodules are painless, in our patient it was painful. The presences of zosteriform skin metastases are rare, and it may be painful and it may mimic true infection locally as present in our case. Although skin metastasis is a sign of inoperable disease in patients with lung cancer, solitary skin metastases can be treated surgically [8-12]. Presence of cutaneous metastases as the initial presentation of lung cancer or appearance during the course of treatment is associated with poor prognosis. Usually, skin metastasis develops after initial diagnosis of the primary malignancy and late in the course of the disease. It is very important to highlight that metastasis may occur at the same time or before the primary cancer has been detected.

\section{Conclusion}

Skin metastasis of lung cancer is rare and carries poor prognosis. It has clinically heterogeneous pattern of presentation in different group of patient may lead to delay in diagnosis and treatment of actual underling cause. Early cutaneous biopsy and search for other possible internal cause of disease is extremely important while managing such type patients [12]. Though, skin metastases from lung cancer are rare, we should suspect it in cases of atypical lesions in the skin in smokers as well as in non-smokers. Skin metastases from lung cancer are a poor prognostic indicator. Early diagnosis of the skin lesions the utmost important in patient presenting with features suggestive of metastatic cutaneous lesion.

\section{References}

1. Lookingbill DP, Spangler N, Helm KF (1993) Cutaneous metastases in patients with metastatic carcinoma: a retrospective study of 4020 patients. J Am Acad Dermatol 29: 228-236.

2. Lee JH, Ahn SJ, Kim HJ, Jang SE, Noh GY, et al. (2011) Cutaneous metastasis from lung cancer: A single-institution retrospective analysis. Tuberc Respir Dis 70: 139-142

3. Hidaka T, Ishii Y, Kitamura S (1996) Clinical features of skin metastasis from lung cancer. Intern Med 35: 459-462.

4. Goljan EF (2006) Rapid review pathology ( $2^{\text {nd }}$ ed). St. Louis, Mosby: 321-324.

5. Song Z, Lin B, Shao L (2012) Cutaneous metastasis as a initial presentation in advanced non-small cell lung cancer and its poor survival prognosis. J Cancer Res Clin Oncol 138: 1613-1617.

6. Mollet TW, Garcia CA, Koester G (2009) Skin metastases from lung cancer Dermatol Online J 5: 1.

7. Ambrogi V, Nofroni I, Tonini G, Mineo TC (2001) Skin metastasis in lung cancer: Analysis of a 10-year experience. Oncol Rep 8: 57-61.

8. Ahmed I (2003) Cutaneous metastases. Dermatol Spain, Mosby: 1953-1956.

9. Rosen T (1980) Cutaneous metastases. Med Clin North Am 64: 885-900.

10. Homler HJ, Goetz CS, Weisenburger DD (1986) Lymphangitic cutaneous metastases from lung cancer mimicking cellulitis. Carcinoma erysipeloides. West J Med 144: 610-612.

11. Kikuchi Y, Matsuyama A, Nomura K (2001) Zosteriform metastatic skin cancer Report of three cases and review of the literature. Dermatol 202: 336-338.

12. Marcoval J, Moreno A, Peyri J (2007) Cutaneous infiltration by cancer. J Am Acad Dermatol 57: 577-580. 